Research Article

\title{
Criteria in Nuclear Fréchet Spaces and Silva Spaces with Refinement of the Cannon-Whittaker Theory
}

\author{
Mahmoud Abul-Ez ${ }^{1}$ and Mohra Zayed (iD) ${ }^{2}$ \\ ${ }^{1}$ Mathematics Department, Faculty of Science, Sohag University, Sohag 82524, Egypt \\ ${ }^{2}$ Mathematics Department, College of Science, King Khalid University, Abha, Saudi Arabia \\ Correspondence should be addressed to Mohra Zayed; mzayed@kku.edu.sa
}

Received 22 September 2020; Revised 18 October 2020; Accepted 20 October 2020; Published 7 November 2020

Academic Editor: Bipan Hazarika

Copyright (c) 2020 Mahmoud Abul-Ez and Mohra Zayed. This is an open access article distributed under the Creative Commons Attribution License, which permits unrestricted use, distribution, and reproduction in any medium, provided the original work is properly cited.

\begin{abstract}
Along with the theory of bases in function spaces, the existence of a basis is not always guaranteed. The class of power series spaces contains many classical function spaces, and it is of interest to look for a criterion for this class to ensure the existence of bases which can be expressed in an easier form than in the classical case given by Cannon or even by Newns. In this article, a functional analytical method is provided to determine a criterion for basis transforms in nuclear Fréchet spaces ((NF)-spaces), which is indeed a refinement and a generalization of those given in this concern through the theory of Whittaker on polynomial bases. The provided results are supported by illustrative examples. Then, we give the necessary and sufficient conditions for the existence of bases in Silva spaces. Moreover, a nuclearity criterion is given for Silva spaces with bases. Subsequently, we show that the presented results refine and generalize the fundamental theory of Cannon-Whittaker on the effectiveness property in the sense of infinite matrices.
\end{abstract}

\section{Introduction}

The existence of bases is one of the fundamental problems in the classical theory of analytic functions. A functional analytic approach to the theory of bases in function spaces emerges naturally when studying classes of functions which play a vital role in applied mathematics and mathematical physics. This paper is entirely devoted to the study of bases in the spaces of holomorphic functions in one or two complex variables. Let us consider two important problems which arise in the study of function spaces as follows:

(1) Does the space under consideration possess a basis?

(2) If this is the case, how can any other basis of this space be characterized?

Assume these problems are answered in a positive way. Then, when $E$ denotes the space and $\left(x_{n}\right)_{n \in \mathbb{N}}$ stands for a basis in $E$, each element $x \in E$ admits a (unique) decomposi- tion of the form $\sum_{n=1}^{\infty} a_{n}(x) x_{n}$ whereby for each $n \in \mathbb{N}, a_{n}$ is a linear functional on $E$. In practice (e.g., in approximation theory), the choice of a suitable basis is very important.

The present work essentially deals with these two fundamental problems in the case where the considered function spaces admit a set of polynomials as a basis (or in the terminology of Cannon-Whittaker, a basic set of polynomials) [1-5]. Basic examples of such function spaces are given by the space of holomorphic functions in an open disc or the space of analytic functions on a closed disc. Of course, as the theory of holomorphic functions in the plane allows generalizations to higher dimensions, analogous problems may be considered in the corresponding function spaces, see $[2,6-14]$.

Cannon and Whittaker $[5,15-17]$ studied the existence of basic sets of polynomials of one complex variable (as bases) in the classical spaces $\mathcal{O}(B(r))$, and later, many authors considered such problem in the space $\mathcal{O}(\bar{B}(r) \times \bar{B}(r))$, see for example $[2,14,18,19]$. The main tool in their investigations 
is a Cannon criterion, determining whether the considered set of polynomials forms a basis for the space (or in the Whittaker terminology, this set of polynomials is effective). So that by the effectiveness of the basic set of polynomials $\left\{P_{n}(z)\right\}$, $z \in \mathbb{C}$, in the closed ball $\bar{B}(r)$, it means that the set forms a base for the class $E$ of holomorphic functions regular in $\bar{B}(r)$ with norm given by $M(f, r), f \in E$.

In our approach here, we introduce the topic from a different point of views, as the change of basis problem presented in the abstract setting of nuclear Fréchet spaces ((NF)-spaces) and nuclear Silva spaces ((NS)-spaces) having in mind essentially the basic example $\mathcal{O}(B(r))$. Criteria are obtained which tell us under which conditions an infinite matrix $P$ is a basis transform in both a (NF)-space and a NS-space with basis. These criteria are then applied to the case of power series spaces, thus yielding a refinement of Cannon's criterion in the case $\mathcal{O}(\bar{B}(r))$ or even those given in $[4,13,18,20]$. It is worth mentioning that this problem has been treated by many authors from different angles for which we may mention $[4,5,21,22]$. Furthermore, significant advances of the subject in higher dimensional spaces have been investigated as in the space of several complex variables $\mathbb{C}^{n}[2]$, monogenic function spaces [8-12, 23, 24], and the matrix function spaces [22]. Much close to the effectiveness problem is the study of spaces of entire functions having finite growth, wherein [25] the author showed that such spaces are also (NF)-spaces.

The main purpose of this paper is to find criteria under which a sequence of vectors $\left(x_{n}\right)_{n \in \mathbb{N}}$ in a (NF)-space $E$ with basis is itself a basis for $E$. This leads to the notion of basispreserving homeomorphisms between (NF)-spaces with bases. The analog study on (NS)-spaces is also provided. Our results are then applied to the case of power series spaces to get a link with the theory of Whittaker on polynomial bases [5] and the theory of Newns who treated the problem of effectiveness by using the topological method approach (see [4]). To begin our investigation, we first give a survey of all necessary definitions and basic results from the general theory of locally convex topological vector spaces and the theory of nuclear spaces. For a more general account, we refer to [26-29]. Then, we point out that the results obtained in this paper might form the starting point of further investigations concerning basis transforms in more general locally convex spaces and higher dimension context.

\section{Preliminaries}

In the sequel, $P=\left(P_{i j}\right), Q=\left(Q_{i j}\right)$, and $T=\left(T_{i j}\right)$ be infinite complex matrices and the infinite identity matrix will be denoted by $I$. All vector spaces will be Fréchet spaces over $\mathbb{C}$, although all results are without changes valid for vector spaces over $\mathbb{R}$. Since we are interested in basis transforms, it will be convenient to look upon a space together with a basis, and notation of the form $(V, b)$ will be used, where $V$ is the vector space and $b=\left\{b_{n}\right\}_{n=1}^{+\infty}$ is a topological basis for $V$. If $V$ and $W$ are isomorphic, then there exists an isomorphism $\varphi$, called a basis-preserving isomorphism, mapping the basis $b$ of $V$ to a basis $c$ of $W$ (i.e., $\varphi\left(b_{n}\right)=c_{n}$ for all $n$ );
$(V, b)$ and $(V, c)$ are called similar. This relation is written $(V, b) \simeq(V, c)$. Obviously, it is not necessarily true that if $b$ and $f$ are two bases for $V$ then $(V, b) \simeq(V, f)$. If it is true however we speak of conjugate bases. If $x=\left(x_{n}\right)$ is a sequence of vectors in $V$ and for each $k$, the series

$$
y_{k}=\sum_{n \in \mathbb{N}} P_{k n} x_{n}, \quad k \in \mathbb{N}
$$

converges, we simply write $y=P x$.

2.1. Köthe Sequence Spaces. Let $\omega$ denote the space of all infinite sequence of complex numbers and let $A=\left(a_{n}^{k}\right)$ be an infinite matrix of real nonnegative numbers such that $a_{n}^{k} \leq a_{n}^{k+1}$ for all $n, k$ and for each $n$, these exist $k$ such that $a_{n}^{k} \neq 0$. Here, $k$ and $n$ stand for the row and the column indices, respectively. With such a matrix $A$, we associate the following subset of $\omega$ :

$$
K(A)=\left\{\xi=\left(\xi_{n}\right) \in \omega:\|\xi\|_{k}=\sum_{n=1}^{\infty} a_{n}^{k}\left|\xi_{n}\right|<+\infty, k \in \mathbb{N}\right\},
$$

with the topology given by the seminorms $\|\cdot\|_{k}$. Note that $\mathscr{P}=\{\|\cdot\|: k \in \mathbb{N}\}$ is a sequence of norms on $K(A)$, which is moreover a system of norms on $K(A)$. Putting for each $n \in \mathbb{N}, e_{n}=\left(\delta_{k n}\right)_{k \in \mathbb{N}}$, we cite the following important result proved by Pietsch in [29].

Theorem 1 (see [29]). $K(A)$ is an (F)-space and $e=\left(e_{n}\right)_{n \in \mathbb{N}}$ is a basis for $K(A)$.

Remark 2. It is well known that if $(V, x)$ is a (NF)-space and the topology is given by seminorms $p_{k}$, then $(V, x)$ $\simeq(K(A), e)$, where $A$ is given by the relation $a_{n}^{k}=p_{k}\left(x_{n}\right)$. This makes it possible to use alternatively the seminorms $p_{k},\|\varphi(.)\|_{k}$, or $|\varphi(.)|_{k}$ where $\varphi$ is the basis-preserving isomorphism.

2.2. Nuclear Fréchet Spaces with Basis. We shall write shortly (F)-space for Fréchet space and (NF)-space for nuclear Fréchet space, as is common in the literature (see [27, 30-32]). Now, let $(E, \mathscr{P})$ be an $(\mathrm{F})$-space where it is henceforth assumed that the countable system of seminorms $\mathscr{P}=\left\{p_{k}\right.$ $: k \in \mathbb{N}\}$ is in fact a sequence of norms. We therefore write $p_{k}=\|\cdot\|_{k}$.

The following result given in $[28,33]$ is useful in the sequel.

Theorem 3 (Banach's homeomorphism theorem). Let $(E, \mathscr{P})$ and $(F, Q)$ be $(F)$-spaces and let $T: E \rightarrow F$ be a bijective and bounded linear operator. Then, $T$ is a homeomorphism and thus $T^{-1}$ is also bounded.

The definition of the (NF)-spaces was introduced in [26] as follows.

Definition 4 ((NF)-space with basis). Let $(E, \mathscr{P})$ be an (F)space. Then, it is called a (NF)-space if for each $k \in \mathbb{N}$, there 
exist $\ell \in \mathbb{N}$ and a sequences $\left(y_{n}\right)_{n \in \mathbb{N}}$ in $E$ and $\left(L_{n}\right)_{n \in \mathbb{N}}$ in $E^{\prime}$ such that for each $x \in E$,

(i) $x=\|_{\|.\|_{k}} \sum_{n \in \mathbb{N}} L_{n}(x) y_{n}$

(ii) $\sum_{n=1}^{\infty}\left\|L_{n}\right\|_{\ell}^{\prime}\left\|y_{n}\right\|_{k}<+\infty$

where $\left\|L_{n}\right\|_{\ell}^{\prime}=\sup _{x \in E,\|x\|_{\ell} \leq 1}\left|L_{n}(x)\right|$ and if $\left\|L_{n}\right\|_{\ell}^{\prime}=\infty$, then $\left\|y_{n}\right\|_{k}=0$

Now, we recall some functional theorems concerning (NF)-spaces with basis.

Theorem 5 (Dynin-Mitiagin [34, 35]). Let (E, x) be a (NF)space with basis. Then, for each $k \in \mathbb{N}$, there exist $\ell \in \mathbb{N}$ and $K_{k}>0$ such that, if for $y \in E, y=\sum_{n=1}^{\infty} \alpha_{n}(y) x_{n}$, where $\alpha_{n}(y)$ is the coefficient function, then

$$
\sum_{n=1}^{\infty}\left|\alpha_{n}(y)\right|\left\|x_{n}\right\|_{k}<K_{k}\|y\|_{\ell}
$$

Corollary 6 (see $[34,35])$. Each base in a (NF)-space with basis is absolute.

Theorem 7 (see [31]). Let E be a (NF)-space with a complete biorthogonal system $\left(x_{n}, x_{n}^{\prime}\right)_{n \in \mathbb{N}}$. Then, $x=\left(x_{n}\right)_{n \in \mathbb{N}}$ is a basis for $E$ if and only if for each $k \in \mathbb{N}$, there exist $\ell \in \mathbb{N}$, and $K_{k}>0$ such that for each $y \in E$,

$$
\sup _{n \in \mathbb{N}}\left|x_{n}^{\prime}(y)\right|\left\|x_{n}\right\|_{k} \leq K_{k}\|y\|_{\ell} .
$$

Theorem 8 (Haslinger's criterion for a (NF)-space with basis $[30])$. Let $(E, x)$ be a (NF)-space with basis and let $\left(y_{n}, y_{n}^{\prime}\right)_{n \in \mathbb{N}}$ be a complete biorthogonal system in E. Then, $\left(y_{n}\right)_{n \in \mathbb{N}}$ is a basis in $E$ if and only if for each $k \in \mathbb{N}$, there exist $\ell \in \mathbb{N}$ and $K_{k}>0$ such that for each $j \in \mathbb{N}$,

$$
\sup _{n \in \mathbb{N}}\left|y^{\prime}{ }_{n}\left(x_{j}\right)\right|\left\|x_{n}\right\|_{k} \leq K_{k}\left\|x_{j}\right\|_{\ell}
$$

2.3. Nuclear Köthe Sequence Spaces. As we have seen that a Köthe sequence space $K(A)$ is an (F)-space with basis $e$, we have also according to [29].

Theorem 9. (see [29]). Let $K(A)$ be a Köthe sequence space. Then, $K(A)$ is nuclear (and hence a (NF)-space with basis) if and only if for each $k \in \mathbb{N}$, there exists $\ell \in \mathbb{N}$ such that

$$
\sum_{n \in \mathbb{N}} \frac{a_{n}^{k}}{a_{n}^{\ell}}<+\infty
$$

Now, suppose that $K(A)$ is a Köthe sequence space and put

$$
\begin{aligned}
& S(A)=\left\{\xi \in \omega: \sup _{n \in \mathbb{N}}\left|\xi_{n}\right| a_{n}^{k}<+\infty\right\}, \\
& \|\xi\|_{k}=\sup _{n \in \mathbb{N}}\left|\xi_{n}\right| a_{n}^{k}, \quad k \in \mathbb{N}, \xi \in S(A) .
\end{aligned}
$$

Then, clearly, $Q=\left\{\|\bullet\|_{k}: k \in \mathbb{N}\right\}$ is a set of norms on $S(A)$. We call $S(A)$ the supremum space associated with $A$. Then, we have

Theorem 10 (see [29]). $(S(A), \mathscr{P})$ is an $(F)$-space.

Remark 11.

(i) Notice that, although $S(A)$ is always an (F)-space, it need not have a basis. Indeed, it is sufficient to consider $A=\left(a_{n}^{k}\right)$ with $a_{n}^{k}=1$ for all $n, k \in \mathbb{N}$

(ii) Notice also that for this matrix $A$, the condition (6) needed for the nuclearity of $K(A)$ is not satisfied. This might suggest that the nuclearity of $K(A)$ could be related to the existence of a basis for $S(A)$. This is indeed the case as it was shown in $[26,30-32,36]$, which can be stated in the following theorem

\section{Theorem 12.}

(i) $K(A)$ is nuclear if and only if $S(A)$ is nuclear

(ii) $K(A)$ and $S(A)$ are homeomorphic

(iii) The standard basis $e=\left(e_{n}\right)_{n \in \mathbb{N}}$ of $K(A)$ is also a basis for $S(A)$, and thus, $S(A)$ is a (NF)-space with basis

This theorem implies immediately that if $K(A)$ is nuclear, then $(K(A), e) \simeq(S(A), e)$.

2.4. Important Remarks. For (F)-spaces, and even for (NF)spaces, the existence of a basis is not always guaranteed, as was shown in $[34,35]$. The Haslinger's criterion mentioned previously in Theorem 8 is essential for our study, namely, to establish whether or not an infinite matrix $P$ determines a basis transform in either (NF)-space or (NS)-space with basis. That is what we strive to achieve in the sequel. This will give a refinement of the criteria of effectiveness problem in the sense of Cannon-Whittaker theory on basis of polynomials.

Beforehand, we give a fruitful study concerning a criterion for an (F)-space to be nuclear showing by supporting examples that the provided criterion is attainable.

\section{A Nuclearity Criterion for (F)-Spaces with Basis}

In this section, we give a criterion stating under which conditions an (F)-space $E$ with basis $x=\left(x_{n}\right)_{n \in \mathbb{N}}$ is nuclear. Let us recall that for an (F)-space $E$ with $x=\left(x_{n}\right)_{n \in \mathbb{N}}$, the associated 
linear functional $x_{n}^{\prime}, n \in \mathbb{N}$ is bounded according to Schauder's theorem [28] which states that "if $E$ is an (F)-space and $\left(x_{n}\right)_{n \in \mathbb{N}}$ is a basis for $E$, then $\left(x_{n}\right)_{n \in \mathbb{N}}$ is a Schauder's basis."

As usual, it is tacitly understood that the topology of the (F)-space is determined by a countable sequence $\left(\|\bullet\|_{m}\right)_{m \in \mathbb{N}}$ of norms.

Theorem 13. Let $E$ be an $(F)$-space with basis $x=\left(x_{n}\right)_{n \in \mathbb{N}}$. Then, the following are equivalent:

(i) $E$ is nuclear

(ii) For each $t \in \mathbb{N}$, there exists $s \in \mathbb{N}$ such that

$$
\sum_{n \in \mathbb{N}} \frac{\left\|x_{n}\right\|_{t}}{\left\|x_{n}\right\|_{s}}<+\infty
$$

Proof. Let $E$ be a nuclear space. Then, by Dynin-Mitiagin theorem 5 , it follows that for all $t \in \mathbb{N}$, there ought to exist $s \in \mathbb{N}$ such that

$$
\sum_{n=0}^{\infty}\left\|x_{n}^{\prime}\right\|_{s}\left\|x_{n}\right\|_{t}<\infty
$$

Since $x_{n}{ }^{\prime}\left(x_{n}\right)=1$, we have $1 \leq\left\|x_{n}{ }^{\prime}\right\|_{s}^{\prime}\left\|x_{n}\right\|_{s}$ or $\left\|x_{n}{ }^{\prime}\right\|_{s}^{\prime} \geq 1 / \|$ $x_{n} \|_{s}$. Consequently,

$$
\sum_{n=0}^{\infty} \frac{\left\|x_{n}\right\|_{t}}{\left\|x_{n}\right\|_{s}}<+\infty
$$

Conversely, assume that for each $t \in \mathbb{N}$, there exists $s \in \mathbb{N}$ such that

$$
\sum_{n=0}^{\infty} \frac{\left\|x_{n}\right\|_{t}}{\left\|x_{n}\right\|_{s}}<+\infty
$$

Let $S$ be the following space:

$$
\begin{aligned}
S & =\left\{a=\left(a_{n}\right)_{n \in \mathbb{N}}: a_{n} \in \mathbb{C} \text { for all } n \in \mathbb{N} \text { and }[a]_{k}\right. \\
& \left.=\sum_{n=0}^{\infty}\left|a_{n}\right|\left\|x_{n}\right\|_{k}<+\infty \text { for all } k \in \mathbb{N}\right\} .
\end{aligned}
$$

Then, clearly, $S$ is a supremum space associated with $A=\left(a_{n}^{k}\right)$ where $a_{n}^{k}=\left\|x_{n}\right\|_{k}$, hence a Fréchet space. By the condition assumed, it follows from Theorem 12 that $S$ is nuclear.

Now, consider the map $\psi: S \rightarrow E$ such that

$$
\psi:\left(a_{n}\right)_{n \in \mathbb{N}} \rightarrow \sum_{n=0}^{\infty} a_{n} x^{n}
$$

Obviously, $\psi$ is linear, continuous, and injective. We now prove that $\psi$ is also surjective, i.e., if $y \in E$ with $y=\sum_{n=0}^{\infty} a_{n} x^{n}$, then $a=\left(a_{n}\right)_{n \in \mathbb{N}} \in S$. Fix $s \in \mathbb{N}$, then as $\sum_{n=0}^{\infty} a_{n} x^{n}$ converges in $F, \lim _{n \rightarrow \infty}\left|a_{n}\right|\left\|x_{n}\right\|_{s}<\infty$ whence

$$
b_{s}=\sup _{n \in \mathbb{N}}\left|a_{n}\right|\left\|x_{n}\right\|_{s}<+\infty
$$

Now, let $t \in \mathbb{N}$ be chosen arbitrarily and let $s \in \mathbb{N}$ such that $\sum_{n=0}^{\infty}\left(\left\|x_{n}\right\|_{t} /\left\|x_{n}\right\|_{s}\right)<+\infty$. Hence,

$$
\sum_{n=0}^{\infty}\left|a_{n}\right|\left\|x_{n}\right\|_{t}=\sum_{n=0}^{\infty}\left|a_{n}\right|\left\|x_{n}\right\|_{s} \frac{\left\|x_{n}\right\|_{t}}{\left\|x_{n}\right\|_{s}} \leq b_{s} \sum_{n=0}^{\infty} \frac{\left\|x_{n}\right\|_{t}}{\left\|x_{n}\right\|_{s}}<+\infty .
$$

Consequently, $a=\left(a_{n}\right)_{n \in \mathbb{N}} \in S$. By virtue of Banach's homeomorphism theorem 3 (see [28]), $\psi$ is bicontentious and so $E$ is homeomorphic to $S$ or in other words $E$ is nuclear.

\section{Remark 14.}

(1) Notice that Theorem 13 provides a relatively simple tool for determining the nuclearity of an (F)-space with basis. In what follows, for an (F)-space $E$ having a basis $\left(x_{n}\right)_{n \in \mathbb{N}}$ satisfying (8), we put

$$
\begin{aligned}
S(A) & =\left\{a=\left(a_{n}\right)_{n \in \mathbb{N}}: a_{n} \in \mathbb{C} \text { and for all } k \in \mathbb{N},[a]_{k}\right. \\
& \left.=\sum_{n=0}^{\infty}\left|a_{n}\right|\left\|x_{n}\right\|_{k}<+\infty\right\}
\end{aligned}
$$

(2) As we have seen, $S(A)$ is a nuclear supremum space which is isomorphic to E. Here, $A=\left(a_{n}^{k}\right)$ with $a_{n}^{k}=$ $\left\|x_{n}\right\|_{k}$

(3) From the isomorphism $\psi$ constructed in the proof above, it follows that if $(E, x)$ is a $(\mathrm{NF})$-space with basis $\left(x_{n}\right)_{n \in \mathbb{N}}$, then the following isomorphisms hold:

$$
(E, x) \simeq(K(A), e) \simeq(S(A), e)
$$

We thus obtain that the natural system of seminorms on $E$ is equivalent to

(i) the system of norms

$$
\|y\|_{k}=\sum_{n \in \mathbb{N}}\left|a_{n}\right| a_{n}^{k}, \quad k \in \mathbb{N}
$$

(ii) the system of norms 


$$
\|y\|_{k}=\sup _{n \in \mathbb{N}}\left|a_{n}\right| a_{n}^{k}, \quad k \in \mathbb{N}
$$

Note that in both cases, $a_{n}=x^{\prime}{ }_{n}(y), n \in \mathbb{N}$.

(4) A nuclearity criterion for the more general case of locally convex spaces having an equicontinuous Schauder basis was proved by Kamthan in [32]. Our Theorem 13 is a special case of his; however, since we are working in (F)-spaces, the proof can be done in a rather easy way. For the case of Köthe spaces, we also refer to [36].

Now, we illustrate the usefulness of the criterion obtained in Theorem 13.

Example 1. Consider the space $\mathcal{O}(B(R))$ of holomorphic functions in the open disk $B(R)$ provided with the countable system $\mathscr{P}$ of seminorms $p_{k}$ where

$$
p_{k}(f)=\sup _{|z| \leq r_{k}}|f(z)|, z \in \mathbb{C},
$$

$\left(r_{k}\right)_{k \in \mathbb{N}}$ being a strictly increasing sequence of positive numbers with $0<r_{k}<R$, and $\lim _{k \rightarrow \infty} r_{k}=R$. One may take, for example, $r_{k}=R-(1 / k)$ (assuming tacitly that $R>1$, if $R \leq 1$, then similar choices may, of course, be made) if $R$ is finite and $r_{k}$ $=k$ if $R$ is infinite.

As we saw before that $(\mathcal{O}(B(R)), \xi)$ is a Fréchet space with basis $\xi=\left(z^{n}\right)_{n \in \mathbb{N}}$, although it is well known that $(\mathscr{O}(B(R)), \xi)$ is a (NF)-space (see [29]), its proof is not trivial.

As will be seen now, the criterion just proved yields the nuclearity of $(\mathcal{O}(B(R)), \xi)$ in an easy way. Indeed, as for each $k \in \mathbb{N}$ and $n \in \mathbb{N}$,

$$
\left\|z^{n}\right\|_{k}=\sup _{|z| \leq r_{k}}\left|z^{n}\right|=r_{k}^{n} .
$$

Taking $k \in \mathbb{N}$ fixed, then for each $\ell>k$, we have

$$
\sum_{n=0}^{\infty} \frac{\left\|z_{n}\right\|_{k}}{\left\|z_{n}\right\|_{\ell}}=\sum_{n=0}^{\infty} \frac{r_{k}^{n}}{r_{\ell}^{n}}=\sum_{n=0}^{\infty}\left(\frac{r_{k}}{r_{\ell}}\right)^{n}<+\infty .
$$

So, the criterion applies.

Remark 15. Notice that, as we previously mentioned,

$$
(\mathcal{O}(B(R)), \xi) \simeq(K(A), e) \simeq(S(A), e),
$$

with $A=\left(a_{n}^{k}\right)$ and $a_{n}^{k}=\sup _{|z| \leq r_{k}}\left|z^{n}\right|$, whence the natural system of seminorms $\mathscr{P}=\left\{\|\bullet\|_{k}: k \in \mathbb{N}\right\}$ and the system of seminorms $\mathscr{P}_{K(A)}$ and $\mathscr{P}_{S(A)}$ induced on $(\mathcal{O}(B(R)), \xi)$ are all equivalent. Here,

$$
\begin{gathered}
\mathscr{P}_{K(A)}=\left\{\|\cdot\|_{k, K}: k \in \mathbb{N}\right\}, \\
\mathscr{P}_{S(A)}=\left\{[\cdot]_{k}: k \in \mathbb{N}\right\},
\end{gathered}
$$

where if $f \in \mathcal{O}(B(R))$ admits the Taylor series at the origin,

$$
f(z)=\sum_{n=0}^{\infty} c_{n} z^{n}
$$

then, for $k \in \mathbb{N},[f]_{k}=\sup _{n \in \mathbb{N}}\left|c_{n}\right| a_{n}^{k}$ and $\|f\|_{k, K}=\sum_{n=0}^{\infty}\left|c_{n}\right| a_{n}^{k}$.

Notice that we already obtain directly, and this by using Cauchy's inequality and the triangle inequality, respectively, the following comparison between the systems of seminorms under consideration for each $k \in \mathbb{N}$ and $f \in \mathcal{O}(B(R)$,

$$
[f]_{k} \leq\|f\|_{k} \leq\|f\|_{k, K} .
$$

Thus, the equivalence between the systems of seminorms established above gives stronger results.

Example 2. Consider the space $\mathcal{O}(\mathrm{B}(\mathrm{R}) \times \mathrm{B}(\mathrm{R}))$ of holomorphic functions in two complex variables in the open polydisc $\mathrm{B}(\mathrm{R}) \times \mathrm{B}(\mathrm{R})$, provided with the countable system $\mathscr{P}$ of seminorms $p_{k}$ where

$$
p_{k}(f)=\sup _{|u| \leq r_{k},|v| \leq r_{k}}|f(u, v)| .
$$

Again, $\left(r_{k}\right)_{k \in \mathbb{N}}$ is strictly increasing sequence of positive numbers with $0<r_{k}<R$ and $\lim _{k \rightarrow \infty} r_{k}=R$. As is well known, $(\mathcal{O}(B(R) \times B(R)), \xi)$ is a Fréchet space. Moreover, as it is shown again by the Taylor series at the origin for any $f \in \mathcal{O}(B(R) \times B(R))$, the sequence $\xi=\left(u^{n} v^{m}\right)_{n, m \in \mathbb{N}}$ is a basis for $\mathcal{O}(B(R) \times B(R))$. Although it is known that $(\mathcal{O}(B(R) \times B(R)), \xi)$ is a (NF)-space, our criterion will yield the nuclearity of it in a very simple way. Indeed, since for each $k \in \mathbb{N}$ and $n, m \in \mathbb{N}$,

$$
\left\|u^{n} v^{m}\right\|_{k}=\sup _{|u| \leq r_{k},|v|<r_{k}}\left|u^{n} v^{m}\right|=r_{k}^{n+m}
$$

we obtain that, taking $k \in \mathbb{N}$ fixed, for each $\ell>k$,

$$
\sum_{n=0}^{\infty} \sum_{m=0}^{\infty} \frac{\left\|u^{n} v^{m}\right\|_{k}}{\left\|u^{n} v^{m}\right\|_{\ell}}=\sum_{n=0}^{\infty} \sum_{m=0}^{\infty}\left(\frac{r_{k}}{r_{\ell}}\right)^{n+m}=\left(\frac{r_{\ell}}{r_{\ell}-r_{k}}\right)^{2}<+\infty,
$$

the nuclearity of this space is proved.

\section{Overview on Basis Transforms in (NF)-Spaces and the General Theory of Effectiveness}

4.1. Basis Transforms and Effectiveness Phenomena. In this section, we provide a general overview of the notion of basis transforms in (NF)-spaces from which a criterion is obtained. This criterion shows that under which conditions, a sequence of vectors $\left(x_{n}\right)_{n \in \mathbb{N}}$ in a (NF)-space $E$ with basis is itself a basis for $E$. This leads to the notion of basis-preserving homeomorphisms between (NF)-spaces with basis. The given results are then applied to the case of power series spaces 
giving a general criterion for basis transforms which improve and refine that one of Cannon-Whittaker on the phenomena of effectiveness.

The idea behind this study is to link the theory of basis transforms in some locally convex space with the theory of Cannon-Whittaker on polynomial bases. In the meantime, we improve and refine the effectiveness phenomena which represents the core of Whittaker's theory.

It is worth mentioning that in the classical treatment of the subject of polynomial bases as was introduced by Whittaker and Cannon [17], the methods for establishing effectiveness depend on the region of effectiveness and on the class of functions for which the base is effective. The first attempt at some uniformity among the different methods was made by Newns, who gave in [4] a topological approach leading to a general theory of effectiveness. Our approach is entirely different depending on functional analytical methods and the basis transforms being performed by means of infinite matrices. In the Cannon-Whittaker theory, the main tool they used depends essentially on assuming the row-finite matrices of coefficients and operators.

In what follows, it is thus understood that $E$ is a (NF)-space with basis $\left(x_{n}, x_{n}^{\prime}\right)_{n \in \mathbb{N}}$. Moreover, $P=\left(P_{i j}\right)$ and $Q=\left(Q_{i j}\right)$ stand for infinite matrices over $\mathbb{C}$ and we denote the infinite identity matrix by $I$. In such a way, we formally write

$$
y_{k}=\sum_{n \in \mathbb{N}} P_{k n} x_{n}, \quad k \in \mathbb{N}
$$

We start by stating the following result by Cnops and Abul-Ez [37].

Theorem 16 (uniqueness theorem). Suppose that $\left(y_{k}\right)_{k \in \mathbb{N}}$ is a basis for E. Then, $P$ has two-sided inverse $Q$.

The following problem now arises: let $E$ be a (NF)-space with basis $\left(x_{n}\right)_{n \in \mathbb{N}}$ and $\left(y_{n}\right)_{n \in \mathbb{N}}$ be a sequence in $E$ satisfying the relations

$$
x_{n}=\sum_{j \in \mathbb{N}} Q_{n j} y_{j}
$$

and formally,

$$
y_{k}=\sum_{n \in \mathbb{N}} P_{k n}\left(\sum_{j \in \mathbb{N}} Q_{n j} y_{j}\right) .
$$

Under which condition on the matrices $P$ and $Q$ we conclude that $\left(y_{k}\right)_{k \in \mathbb{N}}$ is a basis for $E$ ?

Remark 17. In fact, (31) and (32) do not assume that $\left(y_{k}\right)_{k \in \mathbb{N}}$ is a basis. An example is given by Faber polynomials which are valid in a noncircular domain, see [38]. Since the $n$th Faber polynomials is of degree $n$, it is possible to write each $z^{k}$ as a linear combination (even a finite one) of Faber polynomials. However, it is well known that Faber polynomials in general do not form a basis for $\mathcal{O}(B(R))$, but restricted conditions should be considered, see the work of Newns [4]. The answer of the above question is provided by the following interesting results by Cnops and Abul-Ez [37].

Theorem 18 (basis transforms in (NF)-spaces). Let the sequence $\left(y_{k}\right)_{k \in \mathbb{N}}$ in E allow representations of the form (31) and (32). Then, a biorthogonal system $\left(y_{n}, y_{n}^{\prime}\right)_{n \in \mathbb{N}}$ forming a basis for E may be constructed if and only if

(B.1) $P Q=Q P=I$

(B.2) For each $m \in \mathbb{N}$, there exist $\ell \in \mathbb{N}, K_{m}>0$ such that for all $k, n \in \mathbb{N}$

$$
\left|Q_{n k}\right|\left\|y_{k}\right\|_{m} \leq K_{m}\left\|x_{n}\right\|_{\ell}
$$

Remark 19.

(1) From the isomorphism between $\left(E,\left(x_{n}\right)_{n \in \mathbb{N}}\right)$ and $S(A)$ and the derived equivalent system of norms on $E$ (see also Theorems 13 and 12), we have that (B.2) is equivalent to

$\left(\mathrm{B}^{\prime} .2\right)$ For each $m \in \mathbb{N}$, there exist $\ell \in \mathbb{N}$ and $K_{m}>0$ such that for all $n, k, t \in \mathbb{N}$

$$
\left|Q_{n k}\right|\left|P_{k t}\right|\left\|x_{t}\right\|_{m} \leq K_{m}\left\|x_{n}\right\|_{\ell}
$$

(2) On the other hand, using the sum-norm and applying the Dynin-Mitiagin theorem, we deduce that (B.2) and (B.2) ' are equivalent to

$\left(\mathrm{B}^{\prime \prime} .2\right)$ For each $m \in \mathbb{N}$, there exist $\ell \in \mathbb{N}$ and $K_{m}^{\prime}>0$ such that for all $n \in \mathbb{N}$

$$
\sum_{k \in \mathbb{N}} \sum_{t \in \mathbb{N}}\left|Q_{n k}\left\|P_{k t} \mid\right\| x_{t}\left\|_{m} \leq K_{m}^{\prime}\right\| x_{n} \|_{\ell}\right.
$$

(3) Some comments on the requirement that $P$ has twosided inverse $Q$. It is of course possible that $P$ has several two-sided inverses. But, if $P$ is taken to belong to some class of infinite matrices which forms a ring $\mathscr{R}$ under the classical rules of addition and multiplication for matrices and moreover $I \in \mathscr{R}$, then, if $P$ has a two-sided inverses $Q \in \mathscr{R}, Q$ is unique

An example of such a ring $\mathscr{R}$ is the ring $\mathscr{R}_{\text {up }}$ of all infinite upper-triangular matrices or the ring $\mathscr{R}_{\mathrm{lo}}$ of all infinite lower-triangular matrices. It is clear that if, e.g., $P \in \mathscr{R}_{\text {up }}$ has all diagonal elements different from zero, then $P$ has a two-sided inverse $Q$ in $\mathscr{R}_{\text {up }}$ which is therefore unique (see [39]). Nevertheless, it may happen that although the matrix $P$ we are considering belongs to some ring $\mathscr{R}$ of infinite matrices, $P$ admits a two-sided inverse which does not belong 
to the ring $\mathscr{R}$. We address this phenomenon in the following example.

Example 3. Let $\mathscr{R}$ be the ring of all infinite row-finite matrices and $P \in \mathscr{R}$ given by $P=\left(P_{i j}\right)_{i, j=1}^{\infty}$, where

$$
\begin{gathered}
P_{i i}=1, \quad i \in \mathbb{N}, \\
P_{i, i+1}=-1, \quad i \in \mathbb{N}, \\
P_{i j}=0, \quad j \neq i \text { or } j \neq i+1,
\end{gathered}
$$

that is,

$$
P=\left(\begin{array}{cccccc}
1 & -1 & 0 & \cdots & 0 & 0 \\
0 & 1 & -1 & \cdots & 0 & 0 \\
0 & 0 & 1 & -1 & \cdots & 0 \\
: & : & \ddots & \ddots & \ddots & : \\
0 & 0 & \cdots & 0 & 1 & -1 \\
0 & 0 & \cdots & 0 & 0 & 1
\end{array}\right) .
$$

Then, $P$ admits the two-sided inverse $Q=\left(Q_{i j}\right)_{i, j=1}^{\infty}$ with

$$
Q_{i j}=\left(\begin{array}{ll}
0 & \text { if } j<i, \\
1 & \text { if } j \geq i
\end{array}\right.
$$

that is,

$$
Q=\left(\begin{array}{cccccc}
1 & 1 & 1 & \cdots & 1 & 1 \\
0 & 1 & 1 & \cdots & 1 & 1 \\
0 & 0 & 1 & \cdots & 1 & 1 \\
: & : & \ddots & \ddots & : & : \\
0 & 0 & \cdots & 0 & 1 & 1 \\
0 & 0 & \cdots & 0 & 0 & 1
\end{array}\right) .
$$

Clearly, $Q$ is not row-finite.

Remark 20. Applying $P$ to the space $(\mathcal{O}(B(1 / 2)), \xi), P \xi$ is given by

$$
p_{k}(z)=z^{k}-z^{k+1}, \quad k=0,1,2, \cdots,
$$

and using our criterion for a basis transform, $p=P \xi$ is indeed a basis for $\mathcal{O}(B(1 / 2))$.

These considerations therefore suggest that, when an infinite matrix $P$ is given and we wish to use it as a basis transform in some (NF)-space $(E, x)$, we check the following: (i) The existence of an infinite matrix $Q$ which is a twosided inverse of $P$, i.e., (B.1)

(ii) The criterion (B.2) or $\left(\mathrm{B}^{\prime} .2\right)$ or $\left(\mathrm{B}^{\prime \prime} .2\right)$ holds

Suppose (i) is fulfilled. Then, the following situations may occur:

(i.1) $Q$ is the unique two-sided inverse of $P$; then, (ii) above should be verified.

(i.2) $P$ admits several two-sided inverses. If $P$ defines a basis transform, there is a unique two-sided inverse $Q$ such that $Q p$ exists and $Q$ satisfies (B.2). If $P$ does not define a basis transform, the set of inverses

$$
\{Q: Q P=P Q=I \text { and } Q P \text { exists }\}
$$

The above remark leads to the following result.

Corollary 21. Let $P$ be an infinite matrix having a two-sided inverse $Q$ such that $Q p$ converges. Then, $P$ defines a basis transform if and only if the pair $(P, Q)$ satisfies conditions (B.2) (equivalently $\left(B^{\prime} .2\right)$ or $\left.\left(B^{\prime \prime} .2\right)\right)$.

4.2. Power Series Spaces. A class of Köthe sequence spaces which will be of special interest to us is that of so-called power series spaces. Let $\alpha=\left(\alpha_{n}\right)_{n \in \mathbb{N}}$ be an increasing sequence of positive numbers and put for $0<R<+\infty$ fixed,

$$
a_{k}^{n}=\left(R e^{-1 / k}\right)^{\alpha_{n}}
$$

Then, calling $A=\left(a_{n}^{k}\right)$ and associating with it the Köthe sequence space $K(A)$, it is well known that

$$
\text { " } K(A) \text { is nuclear } \Leftrightarrow \lim _{n \rightarrow \infty} \frac{\log n}{\alpha_{n}}=0 .
$$

In the case $R=\infty$, we put

$$
a_{n}^{k}=e^{k \alpha_{n}}
$$

and $A=\left(a_{n}^{k}\right)$. It is then well known that

$$
\text { "K(A) is nuclear } \Leftrightarrow \limsup _{n \rightarrow \infty} \frac{\log n}{\alpha_{n}}<+\infty .
$$

For an account of these results, we refer to [26]. In the case $0<R<+\infty$, the space $K(A)$ is denoted by $K(A)=\Lambda_{R}($ $\alpha$ ) while the case $R=\infty$, the notation $K(A)=\Lambda_{\infty}(\alpha)$ is used.

Notice that, putting $r_{k}=R e^{-1 / k}(0<R<+\infty)$ or $r_{k}=e^{k \alpha_{n}}$ $(R=\infty)$, then $r_{k} \uparrow R$ or $r_{k} \uparrow \infty$.

The question now arises to determine whether or not a given (NF)-space with basis is similar to a space of the type $\left(\Lambda_{R}(\alpha), e\right)$ or $\left(\Lambda_{\infty}(\alpha), e\right)$, the latter spaces being called power series spaces.

Now, we illustrate the problem by means of the following. 
Example 4. The space $(\mathcal{O}(B(R)), \xi), 0<R<+\infty$. Define for each $k \in \mathbb{N}$ and $f \in \mathcal{O}(B(R))$,

$$
\|f\|_{k}=\sup _{|z| \leq R e^{-1 / k}}|f(z)|
$$

As $\lim _{n \rightarrow \infty}(\log n / n)=0$, we can take $\alpha=\left(\alpha_{n}\right)_{n \in \mathbb{N}}$ with $\alpha_{n}=n$ for all $n \in \mathbb{N}$. Consequently, $(\mathcal{O}(B(R)), \xi) \simeq \Lambda_{R}(\mathbb{N}, e)$.

Example 5 (the space $(\mathcal{O}(B(\mathbb{C}), \xi))$. In this case $R=\infty$ and we may describe the topology on $\mathcal{O}(\mathbb{C})$ by the system of seminorms $\mathscr{P}=\left\{\|\bullet\|_{k}: k \in \mathbb{N}\right\}$ where for each $k \in \mathbb{N}$ and $f \in \mathcal{O}(\mathbb{C})$,

$$
\|f\|_{k}=\sup _{|z| \leq e^{k}}|f(z)| .
$$

Hence, for each $n \in \mathbb{N},\left\|z^{n}\right\|_{k}=\sup _{|z| \leq e^{k}}\left|z^{n}\right|=e^{n k}$.

As $\sup _{n \in \mathbb{N}}(\log n / n)<+\infty$, we can take $\alpha=\left(\alpha_{n}\right)_{n \in \mathbb{N}}$ with $\alpha_{n}=n$ for all $n \in \mathbb{N}$. Consequently, $(\mathcal{O}(\mathbb{C}), \xi) \simeq \Lambda_{\infty}(\mathbb{N}, e)$.

In the sequel, we denote for convenience $\left(\Lambda_{R}(\alpha), e\right) \simeq K$ $(\alpha, R)$, the associated supremum space will be denoted by $S$ $(\alpha, R)$.

4.3. Similarity Theorem. The following criterion will completely answer the equation we mentioned above, namely, to know under which conditions a (NF)-space with basis is similar to some power series space $K(\alpha, R)$. To reach this end, let us introduce the following notations.

Given the basis $x=\left(x_{n}\right)_{n \in \mathbb{N}}$ of $E$ and the sequence $\alpha=$ $\left(\alpha_{n}\right)_{n \in \mathbb{N}}$ determining $K(\alpha, R)$, we put for each $k \in \mathbb{N}$ $R(k)=\limsup _{n \rightarrow \infty}\left\|x_{n}\right\|_{k}^{1 / \alpha_{n}}$ and $r(k)=\liminf _{n \rightarrow \infty}\left\|x_{n}\right\|_{k}^{1 / \alpha_{n}}$. Clearly, $r(k) \leq R(k)$ for each $k \in \mathbb{N}$.

Suppose that $R$ is finite (the case $R=+\infty$ is treated in a similar way).

Theorem 22 (similarity theorem basis [37]). Let $(E, x)$ be a (NF)-space with basis and let $K(\alpha, R)$ be some power series space. Then, $(E, x) \simeq K(\alpha, R)$ if and only if

(i) $r(k) \uparrow R$

(iii) $R(k)<R$ for each $k \in \mathbb{N}$

After having established which (NF)-spaces with basis are similar to a power sequence space (Theorem 22), we now aim to apply the criterion obtained for basis transforms (Theorem 18) to the case of power sequence spaces. To do this, consider the power sequence space $K(\alpha, R)$ with basis $e=\left(e_{t}\right)_{t \in \mathbb{N}}$. Then, we know that for each $m \in \mathbb{N}$,

$$
\left\|e_{t}\right\|_{m}= \begin{cases}\left(R e^{-1 / m}\right)^{\alpha_{t}} & \text { for } 0<R<\infty \\ \left(e^{m}\right)^{\alpha_{t}} & \text { for } R=\infty\end{cases}
$$

In the sequel, we put $r_{m}=R^{-1 / m}(0<R<+\infty)$ or $r_{m}=e^{m}$. So, if $P$ is an infinite matrix acting on $e$, then by a direct trans- lation of Theorem 18, the authors of [37] established the following fundamental results.

Theorem 23 (basis criterion). Let $K(\alpha, R)$ be a power sequence space and let $P$ be an infinite matrix. Then, $P e$ is a basis for $K(\alpha, R)$ if and only if

(i) there exists an infinite matrix $Q$ such that $P Q=$ $Q P=I$

(ii) for each $m \in \mathbb{N}$, there exist $\ell \in \mathbb{N}$ and $K_{m}>0$ such that for all $n, k, t \in \mathbb{N}$,

$$
\left|Q_{n k}\right|\left|P_{k t}\right| r_{m}^{\alpha_{t}} \leq K_{m} r_{\ell}^{\alpha_{n}}
$$

Definition 24 (see [37]). Let $P$ be an infinite matrix which has two-sided inverse $Q$ and let $r>0$ be fixed. Then, for $n \in \mathbb{N}$, we put

$$
J_{n}(P, r)=\sup _{k, t}\left|Q_{n k}\right|\left|P_{k t}\right| r^{\alpha_{t}}
$$

and call

$$
J(P, r)=\lim _{n \rightarrow \infty}\left(J_{n}(P, r)\right)^{1 / \alpha_{n}}
$$

if all $J_{n}(P, r)$ are finite, and $+\infty$ otherwise.

From Theorem 23, the following result was deduced in [37].

Theorem 25 (criterion for basis transforms [37]). Let $K(\alpha, R)$ be a power sequence space and let $P$ be an infinite matrix with two-sided inverse $Q$. Then, $P$ determines a basis transform in $K(\alpha, R)$ if and only if for all $0<r<R$,

$$
J(P, r)<R
$$

Remark 26. We saw before that the criterion ( $\left.\mathrm{B}^{\prime} .2\right)$ is equivalent to $\left(\mathrm{B}^{\prime \prime} .2\right)$. This of course prompts the introduction of the following entities: given $r>0$, we put

$$
\begin{aligned}
& Z_{n}(P, r)=\sum_{k} \sum_{t}\left|Q_{n k}\right|\left|P_{k t}\right| r^{\alpha_{t}}, \\
& Z(P, r)=\lim _{n \rightarrow \infty}\left(Z_{n}(P, r)\right)^{1 / \alpha_{n}} .
\end{aligned}
$$

This leads to the following important result which was shown in [37].

Theorem 27 (general criterion for basis transforms [37]). Let $K(\alpha, R)$ be a power sequence space and let $P$ be an infinite matrix having a two-sided inverse $Q$. Then, $P$ determines a basis transforms in $K(\alpha, R)$ if and only if for all $0<r<R$,

$$
Z(P, r)<R
$$

Remark 28. It should be noted that Cannon [15] gave a criterion of the form in (54) for certain spaces of holomorphic 
functions, although he did not prove that $P$ defines a basis transform but only that every function can be represented by a series in $P \xi$.

4.4. Cannon-Whittaker's Criterion Revisited. Consider the space $(\mathcal{O}(B(R)), \xi)$. In [15], Cannon considered an infinite matrix $P$ of the following type:

(i) $P$ is row-finite

(ii) $P$ possesses a row-finite two-sided inverses $Q[5]$

For each $0<r<R$, the following entities were introduced [40]:

The Cannon sum $\lambda_{n}(P, r) \leq \sum_{k, r}\left|Q_{n k}\right|\left\|P_{k}\right\|_{r}$ for the Cannon bases. As for the non-Cannon base (general base), it introduced the Cannon sum in the form:

$$
F_{n}(P, r)=\sup _{t_{1}, t_{2}} \sup _{|z| \leq r} \sum_{k=t_{1}}^{t_{2}} Q_{n k}\left(\sum_{k} P_{k s} z^{s}\right) .
$$

Consequently, for the corresponding Cannon functions

$$
\begin{aligned}
& \lambda(P, r)=\limsup _{n \rightarrow \infty}\left[\lambda_{n}(P, r)\right]^{1 / n}, \\
& \kappa(P, r)=\limsup _{n \rightarrow \infty}\left[F_{n}(P, r)\right]^{1 / n} .
\end{aligned}
$$

4.5. A Refinement of Cannon-Whittaker Criterion for Effectiveness. Cannon proved that the set of polynomials determined by $P \xi$ is effective in $|z|<R$ if and only if $\kappa(P, r)$ $<R$ for all $0<r<R$ and that for Cannon sets $\lambda(P, r)=\kappa(P$, $r)$. When we look at $J_{n}(P, r)$ in the case under consideration, it is clear that for all $n \in \mathbb{N}$ and $0<r<R$,

$$
\begin{gathered}
J_{n}(P, r) \leq F_{n}(P, r) \leq \lambda_{n}(P, r), \\
J(P, r) \leq \kappa(P, r) \leq \lambda(P, r) .
\end{gathered}
$$

Besides the fact that Cannon only proved the effectiveness of the set $P \xi$ under the condition $\kappa(P, r)<R$ for all $0<$ $r<R$ while we pointed out that $J(P, r)<R$ for all $0<r<R$ implies that $P \xi$ is a basis, it obviously follows from $\lambda(P, r)$ $<R$ for all $0<r<R$ that $J(P, r)<R$ for all $0<r<R$. Hence, the given condition here using $J(P, r)$ is weaker than the one obtained by Cannon using $\lambda(P, r)$ in the case of socalled Cannon sets (i.e., sets $P e$ for which $\lambda(P, r)=\kappa(P, r)$ ). There is even more to say. Since we can also use $Z(P, r)$ to establish whether or not $P \xi$ is a basis for $\mathcal{O}(B(R))$ and since clearly for all $n \in \mathbb{N}$ and $0<r<R$,

$$
J_{n}(P, r) \leq F_{n}(P, r) \leq \lambda_{n}(P, r) \leq Z_{n}(P, r),
$$

whence

$$
J(P, r) \leq \kappa(P, r) \leq \lambda(P, r) \leq Z(P, r)
$$

We may conclude that $\lambda(P, r)$ may also be used in the case of non-Cannon sets. Of course, it should also be stressed that the matrices $P$ we are considering need not be row-finite.
Let us illustrate the previous observations in the case of $(\mathcal{O}(B(1)), \xi)$, the space of holomorphic functions in the unit ball.

Example 6. Consider the function $(1-z)^{-1}=\sum_{j=0}^{\infty} z^{j} \in \mathcal{O}$ $(B(1))$. Then, we claim that set $\left\{1 /(1-z), z, z^{2}, \cdots\right.$, $\left.z^{n}, \cdots\right\}$ is a basis for $\mathcal{O}(B(1))$. Indeed, the associated infinite matrix $P$ is given by

$$
P=\left(\begin{array}{cccccc}
1 & 1 & 1 & \cdots & 1 & 1 \\
0 & 1 & 0 & 0 & \cdots & 0 \\
0 & 0 & 1 & 0 & \cdots & 0 \\
: & : & \ddots & \ddots & 0 & : \\
0 & \cdots & 0 & 0 & 1 & 0 \\
0 & \cdots & 0 & 0 & 0 & 1
\end{array}\right) .
$$

Note that $P$ is upper-triangular and has diagonal elements 1. Hence, it possesses a two-sided inverse $Q$ given by

$$
Q=\left(\begin{array}{cccccc}
1 & -1 & -1 & \cdots & -1 & -1 \\
0 & 1 & 0 & 0 & \cdots & 0 \\
0 & 0 & 1 & 0 & \cdots & 0 \\
: & : & \ddots & \ddots & \cdots & : \\
0 & \cdots & 0 & 0 & 1 & 0 \\
0 & \cdots & 0 & 0 & 0 & 1
\end{array}\right) .
$$

Taking $\alpha=\left(\alpha_{n}\right)=\mathbb{N}$, a straightforward calculation yields for each $0<r<1$ that

(i) $J_{0}(P, r)=\sup _{t \in \mathbb{N}, t \geq 1} r^{t}=1$

(ii) $\mathrm{J}_{n}(P, r)=r^{n}, n \geq 1$

Consequently, $J(P, r)=\limsup _{n \rightarrow \infty}\left[J_{n}(P, r)\right]^{1 / n}=1$, so that $J(P, r)<1$ for each $0<r<1$. Hence, $P \xi$ is a basis for the space $\mathcal{O}(B(1))$.

\section{Application: Chebychev Polynomials}

As an actual application of the criterion of basis transforms which refines the analog one of Cannon-Whittaker, we deal with the set of Chebychev polynomials of the first kind (see [41]).

Consider the Chebychev polynomials of the first kind, namely,

$$
P_{n}(z)=\sum_{k=0}^{[n / 1]}\left(\begin{array}{c}
n \\
2 k
\end{array}\right) z^{n-2 k}\left(z^{2}-1\right)^{k}
$$


This set $\left\{P_{n}(z)\right\}_{n \geq 0}$ forms a basic set in the sense of Whittaker [5] and also in the sense of Theorem 16. In view of the expression (53) and (54) and after having the two matrices $\left|Q_{n k}\right|$ and $\left|P_{k t}\right|$, we may have

$$
Z_{n}(P, r)=\liminf _{n \rightarrow \infty}\left\{\sum_{k=0}^{n} \sum_{j=0}^{n / 2}\left|Q_{n k}\right|\left|P_{k t}\right| r^{k-2 j}\left|r^{2}-1\right|^{j}\right\} .
$$

Applying the well known Stirling's formula $n ! \sim \sqrt{2 \pi n^{n}}$ $e^{-n}$ as $n \rightarrow \infty$ to the combinatorics coefficients

$$
\left(\begin{array}{c}
n \\
2 k
\end{array}\right)=\frac{n !}{(2 k) !(n-2 k) !}
$$

we can show after some calculations that the above series in (64) converges well only for $r \leq 1$. Consequently, $Z(P$, $r) \leq r, \quad r \leq 1$. Hence, $\left\{P_{n}(z)\right\}_{n \geq 0}$ is a basis for $\mathcal{O}(B(1))$ and in the terminology of Cannon-Whittaker is effective in unit disc $B(1)$.

The material given in Sections 3 and 4 opened the door to discuss the basis transforms in further functional spaces. In the following section, we investigate some counterpart results in nuclear Silva spaces with basis.

\section{Nuclear Silva Spaces with Basis}

For Fréchet spaces, and even for nuclear Fréchet spaces, the existence of a basis is not always guaranteed, as shown in the fundamental paper by Mitiagin-Zobin and Mitiagin [34, 35]. This problem had been treated by Cnops and Abul-Ez [37] and has been exhaustive demonstrated in Sections 3 and 4 above, where nuclearity criterion is given for Fréchet spaces with basis, as well as basis transforms in such spaces. Related aspects had been treated for the space of holomorphic functions [10] and hyperholomorphic functions [6] to give that the set of Bessel polynomials is a basis in a functional space, and consequently, we have a (NF)-space.

It is worth mentioning that Abul-Ez [25] pointed out that the space of entire functions of finite growth order and type is a (NF)-space, as well as he studied the existence of basis in such space. In this section, we are going to show that if $E$ is a (NF)-space and $F=E^{\prime}{ }_{\beta}$ is the corresponding Silva space, then $F$ admits a basis $\left(x^{\prime}\right)_{k \in \mathbb{N}}$ if and only if $\left(x_{k}^{\prime}\right)_{k \in \mathbb{N}}$ is the dual basis of a basis in $E$. Moreover, a nuclearity criterion is given for a Silva space with basis.

By definition (see [27], p. 264), a Silva space $F$ is the inductive limit of a sequence of Banach spaces $\left(F_{s}\right)_{s \in \mathbb{N}}$ such that for each $s \in \mathbb{N}$, the unit ball of $F_{s}$ is contained in the unit ball of $F_{s+1}$ and is compact in $F_{s+1}$. Several other characterizations of Silva spaces may be given. We mention here the following:

(i) A locally convex space $F$ is a Silva space if and only if $F$ is the strong dual of a Fréchet-Schwartz space $E$. If $\left(\|\cdot\|_{s}\right)_{s \in \mathbb{N}}$ is a defining sequence of seminorms on $E$, then $F$ may also be considered as being the inductive limit of the sequence of Banach spaces $\left(E_{\|\cdot\|_{s}^{\prime}}^{\prime}\right)_{s \in \mathbb{N}}$ whereby for each $s \in \mathbb{N}, E_{\|\cdot\|_{s}^{\prime}}^{\prime}$ is the linear hull of the polar of the closed $\|\cdot\|_{s}$-unit ball (see again [27])

(ii) A locally convex space $F$ is a Silva space if and only if $F$ is a complete DF-Schwartz space in which each null sequence converges locally (see [28], Corollary 12.5.9, and [42])

Moreover, if $F=E_{\beta}^{\prime}$ is a Silva space, $E$ being a FréchetSchwartz space, then $F$ is nuclear if and only if $E$ is nuclear (see, e.g., [28], Theorem 21.5.3).

Now, let $F$ be a Silva space and let $\left(x_{n}\right)_{n \in \mathbb{N}}$ be a basis in $F$. Then, by (ii) and the continuity theorem (see [28], Theorem 14.2.5), $\left(x_{n}\right)_{n \in \mathbb{N}}$ is already a Schauder basis in $F$.

\section{Remark 29.}

(1) As an inductive limit of Banach spaces $\left(F_{s}\right)_{s \in \mathbb{N}}$, we have the following

$$
F_{1} \subset F_{2} \subset F_{3} \cdots \subset F_{n} \subset \cdots \subset F
$$

and we can describe the topology on $F$ by the norms $\|.\|_{s}^{\prime}$ of the spaces $F_{s}$. This means that $\|\cdot\|_{s}^{\prime}$ is defined on $F_{s}$ for $x \in$ $F_{s}:\|x\|_{s}^{\prime} \leq\|x\|_{s+1}^{\prime}$.

In Silva spaces, we have the following criterion of convergence $x_{n} \rightarrow x$ if and only if

(a) there exist $s$ such that $x_{n} \in F_{s}$ for all $n$ and $x \in F_{s}$

(b) $x_{n} \rightarrow\|\cdot\|_{s}^{\prime} x$

(2) Every nuclear Fréchet space is a Fréchet-Schwartz space so the dual of such spaces are Silva spaces

(3) The spaces $\mathcal{O}(\Omega)$ of functions holomorphic (regular) in an arbitrary neighborhood of a compact set $\Omega$ are of this type. Since we are going to prove that having a basis in $F$ is equivalent to having a basis in $F^{\prime}$, we can transfer the basis criterion for nuclear Fréchet spaces to nuclear Silva spaces

\section{Series Representation in Silva Spaces}

As a Silva space is an $F_{+}$-space in the sense of Newns, Theorem 3.2 of [4] and part of its proof may be reformulated as follows.

Theorem 30 (see [4]). Let $F=\mathscr{L}_{s \in \mathbb{N}} F_{s}$ be a Silva space and let $\left(x_{n}\right)_{n \in \mathbb{N}}$ be a sequence in $F$ with $x_{n} \neq 0$ for all $n \in \mathbb{N}$. For $s \in \mathbb{N}$ fixed, suppose that each $x \in F_{s}$ is represented in $F$ by a series of the form $\sum_{n \in \mathbb{N}} \alpha_{n} x_{n}$. Then, there exists $\sigma \in \mathbb{N}$ (depending only on s) such that for each $x \in F_{s}, x=\sum_{n \in \mathbb{N}} \alpha_{n} x_{n}$ in $F_{\sigma}$. 
Moreover, calling $U_{s}$ the space of sequences $\left(\alpha_{n}\right)_{n \in \mathbb{N}}$ in $\mathbb{C}$ such that the series $\sum_{n \in \mathbb{N} n} \alpha_{n} x_{n}$ converges in $F_{\sigma}$ to some element of $F_{s}$ and putting for each $\left(\alpha_{n}\right)_{n \in \mathbb{N}} \in U_{s}$,

$$
\left\|\left(\alpha_{n}\right)_{n \in \mathbb{N}}\right\|_{U_{s}}=\left\|\sum_{n \in \mathbb{N}} \alpha_{n} x_{n}\right\|_{s}+\max _{\ell, t}\left\|\sum_{k=\ell}^{t} \alpha_{k} x_{k}\right\|_{\sigma},
$$

we have that $\left(U_{s},\|\cdot\|_{U_{s}}\right)$ is a Banach space.

Finally, the linear mapping $u: U_{s} \rightarrow F_{s}$ given by $u$ $\left(\left(\alpha_{n}\right)_{n \in \mathbb{N}}\right)=\sum_{n \in \mathbb{N}} \alpha_{n} x_{n}$ is a continuous surjection.

Now, suppose that $\left(x_{n}\right)_{n \in \mathbb{N}}$ is a basis for the Silva space, then clearly the mapping $u: U_{s} \rightarrow F_{s}$ is a continuous bijection, whence by Banach's homeomorphism theorem 3, $u$ is bicontinuous. This leads to the following:

Theorem 31. Let $F=\mathscr{L}_{s \in \mathbb{N}} F_{s}$ be a Silva space with basis $\left(x_{n}\right)_{n \in \mathbb{N}}$ and let for each $s \in \mathbb{N}, U_{s}$ be defined as in Theorem 30. Then, $U_{s}$ and $F_{s}$ are linearly homeomorphic for all $s \in \mathbb{N}$

Corollary 32. Let $F=\mathscr{L}_{s \in \mathbb{N}} F_{s}$ be a Silva space, and $\left(x_{n}\right)_{n \in \mathbb{N}}$ be a basis for $F$, and let for $s \in \mathbb{N}, \sigma \in \mathbb{N}$ be such that $x \in F_{s}$ admits in $F_{\sigma}$ the expansion $x=\sum_{n \in \mathbb{N}} \alpha_{n} x_{n}$. If the continuous linear functional $\alpha_{n}$ on $F$ is not identically zero on $F_{s}$, i.e., ( $\left.\left.\alpha_{n}\right|_{F_{s}} \neq 0\right)$, then $x_{n} \in F_{\sigma}$.

Proof. Suppose that for some $n \in \mathbb{N},\left.\alpha_{n}\right|_{F_{s}} \neq 0$ and $x_{n} \notin F_{\sigma}$. Then, taking $y \in F_{s}$ such that $\alpha_{n}(y) \neq 0$, we have that, as in $F_{\sigma}, y=\sum_{k \in \mathbb{N}} \alpha_{k} x_{k}$, the partial sums $S_{n}(y)=\sum_{i=1}^{n} \alpha_{i}(y) x_{i}$ and $S_{n-1}(y)=\sum_{i=1}^{n-1} \alpha_{i}(y) x_{i}$ belong to $F_{\sigma}$ whence $\alpha_{n}(y) x_{n}=S_{n}(y)$ $-S_{n-1}(y) \in F_{\sigma}$, a contradiction.

\section{Nuclear Silva Spaces with Basis}

Let $F$ be a nuclear Silva space. Then, as we saw in Section 6, $F=E_{\beta}^{\prime}$ whereby $E$ is a $(\mathrm{NF})$-space and $E \simeq F_{\beta}^{\prime}$. In what follows, we therefore denote a basis for $F$ (if it exists) by $\left(x_{k}^{\prime}\right)_{k \in \mathbb{N}}$ and call $\left(x_{k}\right)_{k \in \mathbb{N}}$ the corresponding biorthogonal sequence in $E$, i.e., $x_{\ell} \in E$ with $x_{\ell}\left(x_{k}^{\prime}\right)=\delta_{\ell k}$ for all $k, \ell \in \mathbb{N}$.

Theorem 33. Let $F=E_{\beta}^{\prime}$ be a (NS)-space and let $\left(x_{k}^{\prime}\right)_{k \in \mathbb{N}}$ be a sequence of nonzero elements in $F$. Then, $\left(x_{k}^{\prime}\right)_{k \in \mathbb{N}}$ is a basis in $F$ if and only if $\left(x_{k}\right)_{k \in \mathbb{N}}$ is a basis in $E$.

Proof. If $\left(x_{k}\right)_{k \in \mathbb{N}}$ is a basis for $E$ then $E$ being a (NF)-space, the sequence $\left(x_{k}^{\prime}\right)_{k \in \mathbb{N}}$ is a basis in $E_{\beta}^{\prime}=F$ (see, e.g., [28], Theorem 21.10.6). Conversely, suppose that $\left(x_{k}^{\prime}\right)_{k \in \mathbb{N}}$ is a basis for $F$. Then, since $\left(x_{k}^{\prime}\right)_{k \in \mathbb{N}}$ is a Schauder basis, the biorthogonal system $\left(x_{k}, x_{k}^{\prime}\right)_{k \in \mathbb{N}}$ exists. We prove that the biorthogonal system $\left(x_{k}, x_{k}^{\prime}\right)_{k \in \mathbb{N}}$ is complete, i.e., $E=\operatorname{Span}\left\{x_{k}^{-}: k \in \mathbb{N}\right\}$.

Indeed, call $L=\operatorname{Span}\left\{x_{k}{ }^{-}: k \in \mathbb{N}\right\}$ and suppose that $L \neq E$. Then, if $x \in E \backslash L$, by the Hahn-Banach theorem, there ought to exist $x^{\prime} \in E^{\prime}$ such that $x^{\prime}(x)=1$ and $x^{\prime}(L)=\{0\}$. But, as $x^{\prime}=\sum_{k=1}^{\infty} x_{k}\left(x^{\prime}\right) x_{k}^{\prime}$ and $x_{k}\left(x^{\prime}\right)=0$ for all $k \in \mathbb{N}, x^{\prime}=0$, thus yielding a contradiction.

Now, we prove that the complete biorthogonal system $\left(x_{k}, x_{k}^{\prime}\right)_{k \in \mathbb{N}}$ satisfies Haslinger's criterion [30], i.e., for all $s \in$ $\mathbb{N}$, there exists $\sigma \in \mathbb{N}$ such that

$$
\sup _{k \in \mathbb{N}}\left\|x_{k}^{\prime}\right\|_{\sigma}^{\prime}\left\|x_{k}\right\|_{s}<+\infty
$$

To this end, take $s \in \mathbb{N}$ fixed. On the one hand, by the representation of seminorms, we have for each $k \in \mathbb{N}$ that

$$
\left\|x_{k}\right\|_{s}=\left\|x_{k}\right\|_{s}^{\prime \prime}=\sup _{\left\|x_{k}^{\prime}\right\|_{s}^{\prime}=1}\left|x_{k}\left(x^{\prime}\right)\right|
$$

On the other hand, in view of Theorem 31, there exists $\sigma \in \mathbb{N}$ and a corresponding space $U_{s}$ such that $U_{s}$ is linearly homeomorphic to $F_{s}$. Denoting again by $u$ this isomorphism, we may thus find $C>0$ such that for all $x^{\prime} \in F_{s}$,

$$
\left\|x_{k}^{\prime}\right\|_{s}^{\prime} \geq \frac{1}{C}\left\|u^{-1}\left(x^{\prime}\right)\right\|_{U_{s}} .
$$

Hence, for all $x^{\prime} \in F_{s}$ and $k \in \mathbb{N}$,

$$
\left\|x_{k}^{\prime}\right\|_{s}^{\prime} \geq \frac{1}{C}\left\|x_{k}\left(x^{\prime}\right) x_{k}^{\prime}\right\|_{\sigma}^{\prime}=\frac{1}{C}\left\|x_{k}\left(x^{\prime}\right)\right\|\left\|x_{k}^{\prime}\right\|_{\sigma^{\prime}}^{\prime}
$$

Consequently,

$$
\sup _{k \in \mathbb{N}}\left\|x_{k}\right\|_{s}\left\|x_{k}^{\prime}\right\|_{\sigma}^{\prime} \leq C
$$

By virtue of Haslinger's criterion (see [30]), $\left(x_{k}\right)_{k \in \mathbb{N}}$ is a basis for $E$.

Remark 34. The preceding theorem shows that if $E$ is a (NF)space, then each basis in $F=E_{\beta}^{\prime}$ is the dual basis of a basis in $E$. In view of [28], Theorem 21.10.6, if $\left(x_{k}\right)$ is absolute, so is $\left(x_{k}^{\prime}\right)$. But every basis in $E_{\beta}^{\prime}$ is absolute, so we obtain that each basis in a (NS)-space $F$ is an absolute basis. This duality also leads to the following.

\section{A Nuclearity Criterion for Silva Spaces with Basis}

As was shown in [32], for a vector space $E$ provided with a system of seminorms $\mathscr{P}$ and having a Schauder basis $\left(x_{k}\right)_{k \in \mathbb{N}}$, the following are equivalent:

(i) $E$ is nuclear

(ii) For each $p \in \mathscr{P}$, there exists $q \in \mathscr{P}$ such that 
In this section, a criterion for the nuclearity of Silva spaces $F$ with basis is proved whereby only the sequence of norms of the defining Banach spaces $F_{s}$ is used. In such a way, a criterion for nuclearity is obtained which avoids the use of the system of seminorms defining the inductive limit topology on $F$.

Theorem 35. Let $F=E_{\beta}^{\prime}$ be a Silva with basis $\left(x_{k}^{\prime}\right)_{k \in \mathbb{N}}$. Then, $F$ is nuclear if and only if for each $s \in \mathbb{N}$, there exists $\sigma \in \mathbb{N}$ such that

$$
\sum_{k \in \mathbb{N}} \frac{\left\|x_{k}^{\prime}\right\|_{\sigma}^{\prime}}{\left\|x_{k}^{\prime}\right\|_{s}^{\prime}}<+\infty
$$

Proof. Let $F$ be nuclear. Then, $E$ is a (NF)-space with basis $\left(x_{k}\right)_{k \in \mathbb{N}}$ (see Theorem 30) whence for each $s \in \mathbb{N}$, there exists $\sigma \in \mathbb{N}$ such that (see, e.g., [32])

$$
\sum_{k \in \mathbb{N}} \frac{\left\|x_{k}\right\|_{s}}{\left\|x_{k}\right\|_{\sigma}}<\infty
$$

Moreover, putting $A=\left(a_{k}^{s}\right)_{k, s \in \mathbb{N}}$ with $a_{k}^{s}=\left\|x_{k}\right\|_{s}, E$ is linearly homeomorphic to the nuclear Köthe sequence space $K$ $(A)$ and so the topology on $E$ is also determined by the defining sequence of norms $[.]_{s}$ with

$$
[x]_{s}=\sum_{k \in \mathbb{N}}\left|x_{k}^{\prime}(x)\right|\left\|x_{k}\right\|_{s}, \quad x \in E
$$

(see, e.g., [26]).

Taking duals, we thus have that

$$
F=E_{\beta}^{\prime} \simeq \mathscr{L}_{s \in \mathbb{N}}\left(E^{\prime},\|\cdot\|_{s}^{\prime}\right) \simeq \mathscr{L}_{t \in \mathbb{N}}\left(E^{\prime},[\cdot]_{t}^{\prime}\right),
$$

whereby for each $t \in \mathbb{N}$ and $x^{\prime}=\left(E^{\prime},[\bullet]_{t}^{\prime}\right)$,

$$
\left[x^{\prime}\right]_{t}^{\prime}=\sup _{k \in \mathbb{N}} \frac{1}{\left\|x_{k}\right\|_{t}}\left|x^{\prime}\left(x_{k}\right)\right|
$$

Notice that in particular for each $k \in \mathbb{N}$,

$$
\left[x_{k}^{\prime}\right]_{t}=\frac{1}{\left\|x_{k}\right\|_{t}} \text {. }
$$

Combining (75) and (79), we thus obtain that for each $t$ $\in \mathbb{N}$, there exists $\tau \in \mathbb{N}$ such that

$$
\sum_{k \in \mathbb{N}} \frac{\left[x_{k}^{\prime}\right]_{\tau}}{\left[x_{k}^{\prime}\right]_{t}}<+\infty
$$

Now, let $s \in \mathbb{N}$ be fixed. Then, there exist $t \in \mathbb{N}$ and $K_{s}^{*}$ $>0$ such that for all $k \in \mathbb{N}$,

$$
K_{s}^{*}\left\|x_{k}^{\prime}\right\|_{s} \geq\left[x_{k}^{\prime}\right]_{t},
$$

while for that $t \in \mathbb{N}$, there ought to exist $\tau \in \mathbb{N}$ such that (80) holds. However, for this $\tau$, there exist $\sigma \in \mathbb{N}$ and $K_{\tau}>$ 0 such that for all $k \in \mathbb{N}$,

$$
K_{\tau}\left[x_{k}^{\prime}\right]_{\tau} \geq\left\|x_{k}^{\prime}\right\|_{\sigma}^{\prime}
$$

Consequently, we obtain that for each $s \in \mathbb{N}$, there exists $\sigma \in \mathbb{N}$ such that (74) holds.

Conversely, suppose that for each $s \in \mathbb{N}$, there exists $\sigma \in$ $\mathbb{N}$ such that (74) holds. Calling for each $k, s \in \mathbb{N}$,

$$
a_{k}^{s}= \begin{cases}\frac{1}{\left\|x_{k}^{\prime}\right\|_{s}^{\prime}} & \text { if } x_{k}^{\prime} \in\left(E^{\prime},\|\cdot\|_{s}^{\prime}\right)=F_{s}, \\ 0 & \text { if } x_{k}^{\prime} \notin\left(E^{\prime},\|\cdot\|_{s}^{\prime}\right)=F_{s},\end{cases}
$$

and putting $A=\left(a_{k}^{s}\right)$, we have that the Köthe sequence space $K(A)$ is a (NF)-space, whence its topology is also determined by the sequence of norms $\|\cdot\|_{s}, s \in \mathbb{N}$, with

$$
\begin{gathered}
\|\xi\|_{s}=\sup _{k \in \mathbb{N}}\left(|\xi| a_{k}^{s}\right), \\
\xi=\left(\xi_{k}\right)_{k \in \mathbb{N}} \in K(A) .
\end{gathered}
$$

Its dual $K(A)^{\prime}$ is thus given by

$K(A)^{\prime}=\left\{y=\left(a_{k}\right)_{k \in \mathbb{N}} \in \omega: \exists s \in \mathbb{N w i t h}[y]_{s}=\sum_{k \in \mathbb{N}} \frac{\left|a_{k}\right|}{a_{k}^{s}}<\infty\right\}$,

and of course, $K(A)_{\beta}^{\prime}$ is a nuclear Silva space.

Now, define $B: F \rightarrow \omega$ by

$$
B\left(\sum_{n \in \mathbb{N}} \alpha_{n}\left(x^{\prime}\right) x_{n}^{\prime}\right)=\left(\alpha_{n}\left(x^{\prime}\right)\right)_{n \in \mathbb{N}} .
$$

Then, we claim that $B(F)=K(A)^{\prime}$.

Indeed, if $y \in K(A)^{\prime}$, then there exists $s \in \mathbb{N}$ such that $\sum_{k \in \mathbb{N}}\left(\left|\alpha_{k}\right| / a_{k}^{s}\right)$ whence $\sum_{k \in \mathbb{N}}\left|\alpha_{k}\right| \|\left. x_{k}^{\prime}\right|_{s} ^{\prime}<\infty$ or $\sum_{k \in \mathbb{N}} \alpha_{k} x_{k}^{\prime}$ converges absolutely in $F_{s}$ and so $\sum_{k \in \mathbb{N}} \alpha_{k} x_{k}^{\prime} \in F$. This implies that $K(A)^{\prime} \subset B(F)$.

Now, let $x^{\prime} \in F$ admit the series representation $x^{\prime}=$ $\sum_{k \in \mathbb{N}} \alpha_{k}\left(x^{\prime}\right) x_{k}^{\prime}$. Then, there ought to exist $s \in \mathbb{N}$ such that $x^{\prime}$ $=\sum_{k \in \mathbb{N}} \alpha_{k}\left(x^{\prime}\right) x_{k}^{\prime} \in F_{s}$, the convergence being valid in $F_{s}$ whence $\sup _{k \in \mathbb{N}}\left|\alpha_{k}\left(x^{\prime}\right)\right|\left\|x_{k}^{\prime}\right\|_{s}^{\prime}=K<\infty$. But for this $s \in \mathbb{N}$, there exists by assumption $\sigma \in \mathbb{N}$ such that (74) holds.

We claim that $\left[B\left(x^{\prime}\right)\right]_{\sigma}$ exists. Indeed,

$$
\left[B\left(x^{\prime}\right)\right]_{\sigma}=\sum_{k \in \mathbb{N}} \frac{\left|\alpha_{k}\left(x^{\prime}\right)\right|}{a_{k}^{\sigma}}=\sum_{k \in \mathbb{N}} \frac{\left|\alpha_{k}\left(x^{\prime}\right)\right|}{a_{k}^{s}} \frac{a_{k}^{s}}{a_{k}^{\sigma}} \leq K \sum_{k \in \mathbb{N}} \frac{a_{k}^{s}}{a_{k}^{\sigma}}<\infty .
$$


Consequently, $B\left(x^{\prime}\right) \in K(A)^{\prime}$ and so $B(F) \subset K(A)^{\prime}$. Obviously, $B$ is an isomorphism between $F$ and $K(A)^{\prime}$.

Now, we show that $B^{-1}$ is continuous. Indeed, take $y \in$ $K(A)^{\prime}$. Then, there exists $s \in \mathbb{N}$ such that

$$
[y]_{s}=\sum_{k \in \mathbb{N}} \frac{\left|\alpha_{k}\left(x^{\prime}\right)\right|}{a_{k}^{s}}<\infty
$$

Putting $x^{\prime}=B^{-1} y$, we have

$$
[y]_{s}=\sum_{k \in \mathbb{N}}\left|\alpha_{k}\right|\left\|x_{k}^{\prime}\right\|_{s}^{\prime} \geq\left\|\sum_{k \in \mathbb{N}} \alpha_{k} x_{k}^{\prime}\right\|_{s}^{\prime}=\left\|x^{\prime}\right\|_{s}^{\prime} .
$$

By virtue of the open mapping theorem, $F$ and $K(A)^{\prime}$ are linearly homeomorphic whence $F$ is nuclear.

Remark 36. Another possible approach to the proof of Theorem 35 is one using the Grothendieck-Pietsch criterion for nuclearity for Köthe sequence spaces (see [29, 36]). Indeed, from our criterion, it follows that the basis considered is absolute and an explicit expression for the system of seminorms defining the topology of $F$ can be given in terms of a Köthe sequence space. Applying the Grothendieck-Pietsch criterion then yields the nuclearity of the space $F$. On the other hand, if $F$ is nuclear and has a basis, it follows from Theorem 33 that this basis is absolute whence $F$ is linearly homeomorphic to some Köthe sequence space $\mathrm{K}(\mathrm{A})$, which, by assumption upon $F$, is nuclear. Hence, the GrothendieckPietsch criterion holds which can then be translated into the criterion of the above Theorem 35. For the GrothendieckPietsch criterion, we refer to ([28], Theorem 21.6.2).

Example 7. In [32], Kamthan introduced the following Fréchet space $\left(\mathcal{O}_{A}(\operatorname{Re} z<A), \zeta\right)$ of holomorphic functions. Let $\lambda=\left(\lambda_{n}\right)_{n \in \mathbb{N}}$ be a fixed strictly increasing sequence of positive real numbers. With each sequence $\left(a_{n}\right)_{n \in \mathbb{N}}$ such that $\lim _{n \rightarrow \infty} \sup \left(\left|a_{n}\right| / \lambda_{n}\right) \leq-A$, we associate the function

$$
f(z)=\sum_{n=1}^{\infty} a_{n} e^{z \lambda_{n}}, \quad z \in \mathbb{C}
$$

Take $\varepsilon>0$ arbitrary chosen and consider the half-plane

$$
A_{\varepsilon}=\{z \in \mathbb{C}: \operatorname{Re} z \leq A-\varepsilon\}
$$

Then, for each $n \in \mathbb{N}$ and $z \in A_{\mathcal{\varepsilon}}$,

$$
\left|a_{n}\right|\left|e^{z \lambda_{n}}\right| \leq\left|a_{n}\right| e^{A \lambda_{n}} e^{-\varepsilon \lambda_{n}}
$$

But, in virtue of the assumption,

$$
\lim _{n \rightarrow \infty} \sup \frac{\log \left|a_{n}\right|}{\lambda_{n}} \leq-A,
$$

we find that

$$
\left|a_{n}\right| e^{A \lambda_{n}}=e^{\log \left|a_{n}\right|+A \lambda}
$$

whence there exist $C>0$ such that

$$
\sup _{n \in \mathbb{N}}\left|a_{n}\right| e^{A \lambda_{n}} \leq C
$$

Consequently,

$$
\sum_{n \in \mathbb{N}} \sup _{z \in A_{\varepsilon}}\left|a_{n} e^{z \lambda_{n}}\right| \leq C \sum_{n \in \mathbb{N}} e^{-\varepsilon \lambda_{n}}<+\infty
$$

whence the series defining $f$ is normally convergent on each $A_{\varepsilon}, \varepsilon>0$. Consequently, $f \in \mathcal{O}_{A}(\operatorname{Re} z<A)$.

We call $\mathcal{O}_{A}(\operatorname{Re} z<A)$ the subspace of $\mathcal{O}(\operatorname{Re} z<A)$ consisting of the elements $f$ just defined and provide $\mathcal{O}_{A}(\mathrm{Re}$ $z<A$ ) with the system $\mathscr{P}$ of seminorms $p_{k}$ with

$$
p_{k}(f)=\sup _{\operatorname{Re} z \leq A-(1 / k)}|f(z)|
$$

Then, it was proved by Kamthan that $\left(\mathcal{O}_{A}(\operatorname{Re} z<A), \mathscr{P}\right)$ is a Fréchet space. From the definition itself of the elements $f$ in $\mathcal{O}_{A}$, it follows that the sequence of functions $\zeta=\left(e^{z \lambda_{n}}\right)_{n \in \mathbb{N}}$ is a basis for the space $\left(\mathscr{O}_{A}(\operatorname{Re} z<A), \mathscr{P}\right)$. Then, we claim that $\left(\mathcal{O}_{A}(\operatorname{Re} z<A), \zeta\right)$ is a $(\mathrm{NF})$-space. Indeed, from the definition itself of $\mathcal{O}_{A}(\operatorname{Re} z<A)$, it follows that $\zeta$ is a basis for it.

Having that $\left(\mathcal{O}_{A}(\operatorname{Re} z<A), \zeta\right)$ is a $(\mathrm{NF})$-space, then using the discussion in Section 6, it can be seen that it is a (NS)space with basis. Now, let $k \in \mathbb{N}$ be fixed and take any $l \in \mathbb{N}$ with $l>k$. Then,

$$
\sum_{n=1}^{\infty} \frac{\left\|e^{z \lambda_{n}}\right\|_{k}}{\left\|e^{z \lambda_{n}}\right\|_{l}}=\sum_{n=1}^{\infty} \frac{e^{(A-(1 / k)) \lambda_{n}}}{e^{(A-(1 / l)) \lambda_{n}}}=\sum_{n=1}^{\infty} e^{((1 / l)-(1 / k)) \lambda_{n}}<\infty
$$

Therefore, by our criterion (Theorem 35), the nuclearity is proved.

Example 8. Consider the space $\left(\mathcal{O}\left(\bar{S}_{R}\right), \xi\right)$ of holomorphic functions in two complex variables $z, w$, provided with the countable system $\mathscr{P}$ of seminorms $p_{k}$ where

$$
p_{k}(f)=\sup _{\bar{S}_{r_{k}}}|f(z, w)| \text {, }
$$

and $\bar{S}_{r}$ is the closed hypersphere defined by

$$
\sup _{\bar{S}_{r_{k}}}=\left\{z, w \in \mathbb{C}:|z|^{2}+|w|^{2} \leq r_{k}^{2}\right\}
$$

Again, $\left(r_{k}\right)_{k \in \mathbb{C}}$ is a strictly increasing sequence of positive numbers with $0<r_{k}<R$ and $\lim _{k \rightarrow \infty} r_{k}=R$. As is well known, $\left(\mathcal{O}\left(\bar{S}_{R}\right), \xi\right)$ is a Fréchet, and consequently, it can be proved that it is a Silva space. Moreover, as it was shown again by the Taylor series at the origin for any $f$ 
$\in \mathcal{O}\left(\bar{S}_{R}\right)$, the sequence

$$
\xi=\left(z^{m} w^{n}\right)_{m, n \in \mathbb{N}}
$$

is a basis for $\mathcal{O}\left(\bar{S}_{R}\right)$. Although it is known that $\left(\mathcal{O}\left(\bar{S}_{R}\right), \xi\right)$ is a (NF)-space and then a (NS)-space, our criterion will yield the nuclearity of it in a very simple way. Indeed, as for each $k, m, n \in \mathbb{N}$, it can be proved that (see [2])

$$
\left\|z^{m} w^{n}\right\|_{k}=\frac{r_{k}^{m+n}}{\sigma_{m, n}}
$$

where $\sigma_{m, n}$ is given by

$$
\sigma_{m, n}= \begin{cases}\frac{(m+n)^{(1 / 2)(m+n)}}{m^{m / 2} n^{n / 2}} & \text { when } m, n>0, \\ 1 & \text { when } m=0 \text { or } n=0 .\end{cases}
$$

Then, we obtain that, taking $k \in \mathbb{N}$ fixed, for each $\ell$ $>K$,

$\sum_{m=0}^{\infty} \sum_{n=0}^{\infty} \frac{\left\|z^{m} w^{n}\right\|_{k}}{\left\|z^{m} w^{n}\right\|_{\ell}}=\sum_{m=0}^{\infty} \sum_{n=0}^{\infty}\left(\frac{r_{k}}{r_{\ell}}\right)^{m+n}=\left(\frac{r_{\ell}}{r_{\ell}-r_{k} r_{\ell}}\right)^{2}<+\infty$,

the nuclearity of this space is proved.

\section{General Remarks and Comments}

The properties of series of the form $\sum_{i=0}^{\infty} c_{i} P_{i}(z), z \in \mathbb{C}$ where $P_{i}(z), i=0,1, \cdots$ are prescribed polynomials and $e_{i}$ chosen in a field $\mathbb{K}$ of scalars, widely differ according to the particular chosen polynomials. For example, the region of convergence (which is called the region of effectiveness) may be a circle (for Taylor series), an ellipse (for series of Legendre polynomials), and a half-plane (for Newton's interpolation series). Whittaker [40], in his attempt to find the common properties exhibited by all these polynomials, introduced the notion of basic sets of polynomials. In his work [5], he defined the basic sets, basic series, and effectiveness of basic sets. In [15-17], Cannon obtained the necessary and sufficient condition for the effectiveness of basic sets for classes of functions of finite radii of regularity and entire functions. In the classical treatment of the subject of basic sets [5], the methods for establishing effectiveness depend on the region of effectiveness and the class of functions for which the set is effective.

The first attempt at some uniformity among the different methods was made by Newns who gave in [4] a topological approach leading to a general theory of effectiveness. It is well known that a lot of classical function spaces are important examples of so-called nuclear Fréchet spaces, for example, spaces of null solutions of elliptic partial differential operators with constant coefficients such as the Cauchy-Riemann operator and the Laplace operator.

On an abstract level, the problem of effectiveness of basic sets of polynomials in spaces of holomorphic functions as introduced by Cannon-Whittaker may be therefore consid- ered as being related to the problem of the change of bases in nuclear Fréchet spaces as well as in other related spaces.

In the present work, we show that general criteria for basis transforms are obtained for the nuclearity of Fréchet spaces with basis which are applied to characterize basis transforms in terms of infinite matrices in classes of nuclear Fréchet spaces. This study is considered to be a refinement of those given by Cannon, Whittaker, and Newns and all relevant generated topics.

In such a way analog results are given concerning nuclear Silva spaces with bases. This might form a starting point for further investigations regarding the basis transforms in more general locally convex spaces or higher dimensional spaces with different domains of convergence. Finally, it will be expected in the forthcoming work to study basis transforms in

(i) spaces involving product bases, inverse bases, transpose bases, derived and integrated bases, etc.

(ii) spaces of entire functions having finite growth

(iii) spaces of holomorphic functions in Faber regions

(iv) spaces of several complex variables

(v) monogenic function spaces in the framework of Clifford analysis.

\section{Data Availability}

The data used to support the findings of this study are included within the article.

\section{Conflicts of Interest}

The authors declare that there is no conflict of interest.

\section{Acknowledgments}

The authors extend their appreciation to the Deanship of Scientific Research at King Khalid University for funding this work through research groups program under grant R.G.P.1/184/41.

\section{References}

[1] M. Nassif, Products and Zeros of Basic Sets, Assiut University Monograph Series 2, Assiut, Egypt, 1958.

[2] M. Nassif, Composite sets of polynomials of several complex variables, Publicationes Mathematicaes Debrecen, 1971.

[3] M. Nassif and R. H. Makar, "On non-algebraic basic sets of polynomials," Indagationes Mathematicae (Proceedings), vol. 58, pp. 120-129, 1955.

[4] W. Newns, "On the representation of analytic functions by infinite series," Philosophical Transactions of the Royal Society of London. Series A, Mathematical and Physical Sciences, vol. 245, no. 900, pp. 429-468, 1953.

[5] J. Whittaker and C. Gattegno, Sur les séries de base de polynbmes quelconques, Gauthier-Villars, Paris, France, 1949.

[6] M. Abdalla, M. Abul-Ez, and J. Morais, "On the construction of generalized monogenic Bessel polynomials," Mathematical 
Methods in the Applied Sciences, vol. 41, no. 18, pp. 9335-9348, 2018.

[7] M. Abul-Ez, "Product simple sets of polynomials in Clifford analysis," Rivista di Matematica della Università di Parma, vol. 5, pp. 283-293, 1994.

[8] M. Abul-Ez and D. Constales, "Basic sets of polynomials in Clifford analysis," Complex Variable, vol. 14, pp. 177-185, 1990.

[9] M. Abul-Ez and D. Constales, "Linear substitution for basic sets of polynomials in Clifford analysis," Portugaliae Math, vol. 48, pp. 143-154, 1990.

[10] M. A. Abul-Ez, "Bessel polynomial expansions in spaces of holomorphic functions," Journal of Mathematical Analysis and Applications, vol. 221, no. 1, pp. 177-190, 1998.

[11] M. Abul-Ez, D. Constales, J. Morais, and M. Zayed, "Hadamard three-hyperballs type theorem and overconvergence of special monogenic simple series," Journal of Mathematical Analysis and Applications, vol. 412, no. 1, pp. 426-434, 2014.

[12] M. Abul-Ez and M. Zayad, "Similar transposed bases of polynomials in Clifford analysis," Applied Mathematics \& Information Sciences, vol. 4, pp. 63-78, 2010.

[13] G. F. Hassan, L. Aloui, and A. Bakali, "Basic sets of special monogenic polynomials in Fréchet modules," Journal of Complex Analysis, vol. 2017, Article ID 2075938, 11 pages, 2017.

[14] W. F. Kumuyi and M. Nassif, "Derived and integrated sets of simple sets of polynomials in two complex variables," Journal of Approximation Theory, vol. 47, no. 4, pp. 270-283, 1986.

[15] B. Cannon, "On the convergence of series of polynomials," Proceedings of the London Mathematical Society, vol. s2-43, no. 1, pp. 348-365, 1938.

[16] B. Cannon, "On convergence properties of basic series," Journal of the London Mathematical Society, vol. 14, pp. 51-62, 1939.

[17] B. Cannon, "On the representation of integral functions by general basic series," Mathematische Zeitschrift, vol. 45, pp. 185-208, 1939.

[18] J. Adepoju and M. Nassif, "Effectiveness of polynomials of two complex variables in polycylinders and in Faber regions," Journal of Natural Sciences and Mathematics, vol. 24, no. 2, pp. 153-172, 1984.

[19] J. Adepoju and A. A. Mogbademu, "Effectiveness of cannon and composite sets of polynomials of two complex variables in Faber regions," Proyecciones (Antofagasta), vol. 39, no. 3, pp. 651-662, 2020.

[20] J. Adepoju, Basic sets of Goncarov polynomials and Faber regions [Ph.D. Thesis], University of Lagos, Lagos, Nigeria, 1979.

[21] R. Makar and B. Makar, "On algebraic simple monic sets of polynomials," Proceedings of the American Mathematical Society, vol. 2, no. 4, pp. 526-537, 1951.

[22] K. Sayyed and M. Abul-Ez, "Linear substitutions for functions and sets of polynomials of two complex matrices," Bulletin of Faculty of Science, Sohag university, vol. 11, no. 1, pp. 45-51, 1982, MR. 85g; 32001.

[23] M. Zayed, "Generalized Hadamard product bases of special monogenic polynomials," Advances in Applied Clifford Algebras, vol. 30, no. 1, 2020.

[24] M. Zayed, "Lower growth of generalized Hadamard product functions in Clifford setting," Bulletin of the Malaysian Mathematical Sciences Society, 2020.
[25] M. Abul-Ez, "A study on the space of entire functions having finite growth," National Academy Science Letters, vol. 25, no. 3-4, pp. 105-114, 2002.

[26] E. Dubinsky, The Structure of Nuclear Fréchet-Spaces, Lecture Notes in Mathematics, Springer-Verlag, Berlin Heidelberg, 1979.

[27] H. G. Garnir, M. De Wilde, and J. Schmets, Analyse Fonctionnelle: Théorie Constructive Des Espaces Linéaires à SemiNormes, Basel, Birkhäuser, Stuttgart, 1968.

[28] H. Jarchow, Locally Convex Spaces, Mathematische Leitfäden, Letfäden, Teubner, Stuttgart, 1981.

[29] A. Pietsch, Nuclear Locally Convex Spaces, Springer, Berlin, 1972.

[30] F. Haslinger, "Complete biorthogonal systems in nuclear (F)spaces," Mathematische Nachrichten, vol. 83, no. 1, pp. 305310, 1978.

[31] F. Haslinger, "Quasibasen und nukleare (F)-Räume," Sitzungsberichte Österreichische Akademie der Wissenschaften Mathematische-naturwissenschaftliche Klasse, vol. 184, pp. 333-338, 1975.

[32] P. K. Kamthan, "A nuclearity criterion for spaces having a Schauder base," Acta Mathematica Vietnamica, vol. 7, pp. 41-45, 1982.

[33] L. Kantorovitch and G. Akilov, Analyse Fonctionnelle, MIR, Moscow, 1981.

[34] B. S. Mitiagin and N. M. Zobin, "Contre-exemple à l'existence d'une base dans un espace de Fréchet nucléaire," CR Acad. Sci. Paris Ser.; A, vol. 279, pp. 255-256, 1974.

[35] B. S. Mitiagin, "Nuclear Fréchet spaces without bases II. The case of strongly finite-dimensional decomposition," Bulletin de l'Académie Polonaise des Sciences, Série des Sciences Mathématiques Astronomiques et Physiques, vol. 24, no. 7, pp. 475480, 1976.

[36] N. J. Kalton, “On absolute bases," Mathematische Annalen, vol. 200, no. 3, pp. 209-225, 1973.

[37] J. Cnops and M. Abul-Ez, "Basis transforms in nuclear Fréchet spaces," Simon Stevin: A Quarterly Journal of Pure and Applied Mathematics, vol. 67, pp. 145-156, 1993.

[38] J. L. Ullman, "Studies in Faber polynomials. I," Transactions of the American Mathematical Society, vol. 94, no. 3, pp. 515528, 1960.

[39] P. Dienes, "Notes on linear equations in infinite matrices," The Quarterly Journal of Mathematics, vol. 1, pp. 253-268, 1932.

[40] J. M. Whittaker, Interpolatory function theory, The University Press, 1935.

[41] J. Mason and D. Handscomb, Chebyshev Polynomials, CRC Press, LLC, 2003.

[42] J. Cooper, "On a generalisation of Silva spaces," Mathematische Annalen, vol. 182, no. 4, pp. 309-313, 1969. 